\title{
Complete low-barrier side-chain route for olefin formation during methanol conversion in H-SAPO-34
}

\author{
Kristof De Wispelaere, Karen Hemelsoet, Michel Waroquier and Veronique Van \\ Speybroeck*
}

Center for Molecular Modeling (CMM), Ghent University, Technologiepark 903, B-9052 Zwijnaarde, Belgium; QCMM-alliance, Ghent-Brussels, Belgium

*Corresponding author: Veronique.VanSpeybroeck@UGent.be

\begin{abstract}
The methanol to olefins process is an alternative for oil-based production of ethene and propene. However, detailed information on the reaction mechanisms of olefin formation in different zeolite is lacking. Herein a first principle kinetic study allows elucidating the importance of a side-chain mechanism during methanol conversion in $\mathrm{H}$ SAPO-34. Starting from the experimentally observed hexamethylbenzene, a full low-barrier catalytic cycle for ethene and propene formation is found. The olefin elimination steps exhibit low free energy barriers due to a subtle interplay between an $\mathrm{sp}^{3}$ carbon center of the organic intermediate, stabilizing non-bonding interactions and assisting water molecules in the zeolite material.
\end{abstract}

Keywords: methanol to olefin, heterogeneous catalysis, side-chain mechanism, zeolites, first principle kinetics, H-SAPO-34 


\section{Introduction}

The methanol to olefins process (MTO), using natural gas, coal or biomass as raw material, is an important alternative to oil-based routes for the production of ethene and propene. Detailed understanding of this complex process and the subtle balance between various parameters is a highly debated topic, as reviewed in recent works.[1-3] MTO conversion occurs in acidic zeolites and zeotype catalysts. H-SAPO-34 - exhibiting the chabazite topology - is industrially preferred due to its high selectivity toward light olefins.[4] The generally accepted reaction mechanism for MTO is based on a hydrocarbon pool (HP) mechanism, meaning that organic molecules trapped within the inorganic zeolite framework act as co-catalysts.[5] For small-pore chabazite catalysts these organic reaction scaffolds consist mainly of methylated aromatics, with hexamethylbenzene (HMB, see Figure 1) showing the highest activity as demonstrated by GC-MS analyses of ${ }^{13} \mathrm{C}$-labeled methanol experiments and computational studies.[6-10] Starting from this compound, aromatic-based cycles have been proposed for olefin formation, in particular the side-chain and paring mechanisms. A comprehensive review on the various reaction mechanisms in MTO catalysis can be found in reference [3]. In the side-chain mechanism an alkyl chain is formed through various side-chain methylations allowing elimination of olefins with regeneration of the original HP species. In the paring mechanism growth of the alkyl chain originates from ring contraction/expansion reactions. Experimentally there are various arguments advocating the side-chain or paring route. During pulse experiments with ${ }^{13} \mathrm{C}$-labeled methanol, carbon label scrambling is observed in the produced olefins and HP species.[7, 9] This exchange between ${ }^{12} \mathrm{C}$ ring carbon atoms and ${ }^{13} \mathrm{C}$ methyl carbon atoms is often believed to be indicative for the occurrence of a paring type mechanism.[9] Some caution regarding this conclusion is warranted since experimentally estimated rates of carbon label incorporation in the ring occur very fast at high temperatures.[11, 12] Furthermore, it is observed that HMB present in H- 
SAPO-34 without methanol feed still produces olefins, which is not readily explained by the side-chain mechanism.[13] Obtaining more insight into the exact mechanism is of utmost importance to design the MTO process at the molecular scale. Therefore, plenty of theoretical studies have been conducted as they can independently study individual reaction cycles. Various studies have advocated the side-chain mechanism,[12, 14, 15] however in every proposed cycle energy barriers of at least $200 \mathrm{~kJ} / \mathrm{mol}$ were present, being too high for a viable catalytic cycle. The bottleneck is mainly the olefin elimination step, for which various reaction mechanisms have been explored. In this work, we propose a full catalytic cycle for the production of ethene and propene in H-SAPO-34 wherein all steps have free energy barriers at $670 \mathrm{~K}$ below $100 \mathrm{~kJ} / \mathrm{mol}$. All conclusions in this work are based on free energies and reaction rate coefficients determined from first principle DFT-D calculations on extended finite clusters, representative for the catalytic environment (Figure S1 of the SI).

\section{Computational Methods}

H-SAPO-34 was represented by an extended 44T cluster containing one acid site (Figure S1 of the SI). DFT calculations have been performed with the Gaussian09 software package.[16] ONIOM(B3LYP/6-31+g(d):PM3) geometries are combined with B3LYP/6$311+\mathrm{g}(\mathrm{d})-\mathrm{D}$ energies. Dispersion is accounted for by means of D3 corrections as proposed by Grimme and co-workers.[17] The B3LYP-D results were validated with the ONIOM(M062X/6-31+g(d):PM3) // M06-2X/6-311+g(d) level of theory. It was shown that with this approach, one can obtain "near chemical accurate" kinetic data.[18] To gain insight into the kinetically relevant steps of the proposed mechanism, it was assumed that in each step all species are adsorbed in the zeotype material. Hence intrinsic free energy barriers and unimolecular rate coefficients are being reported in this work. As such, a comprehensive study of adsorption and desorption is beyond the present scope. However, methodologies 
yielding accurate adsorption enthalpies are available nowadays.[19, 20] Free energies and entropies were obtained with the in-house software package Tamkin.[21] UV-Vis data result from TD-DFT calculations on the optimized ground state geometries using B3LYP/6$31+\mathrm{g}(\mathrm{d})$. All reported gas phase proton affinities are calculated at the B3LYP/6-311+g(d) level of theory, including zero-point energy corrections. However, more accurate proton affinities can be obtained by applying more expensive computational methods as reported in reference [22]. More details on the applied computational methods can be found in the supporting information.

\section{Results and Discussion}

In this research note, we propose a complete catalytic cycle for ethene and propene production in H-SAPO-34 without the occurrence of a single bottleneck, i.e. all steps have free energy barriers at $670 \mathrm{~K}$ below $100 \mathrm{~kJ} / \mathrm{mol}$. The low-barrier olefin elimination reaction is characterized by the following crucial factors (Figure 1): (1) the $\mathrm{sp}^{3}$ hybridization of the ring carbon carrying the side-chain, (2) the formation of an alkyl-benzene stabilized complex in the transition state and (3) the assistance of water during the deprotonation of the alkyl group.

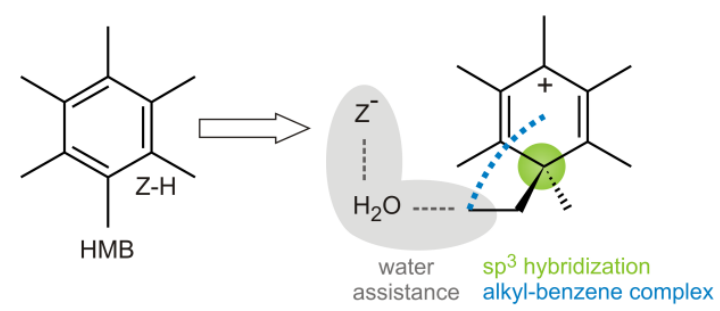

Figure 1. The side-chain route toward ethene formation starting from HMB with indication of the three factors determining the low-barrier ethene split-off.

The full catalytic cycle for ethene and propene formation starting from HMB is shown in Figure 2. A similar cycle can be constructed for the formation of isobutene (Figure S2 of the SI). 


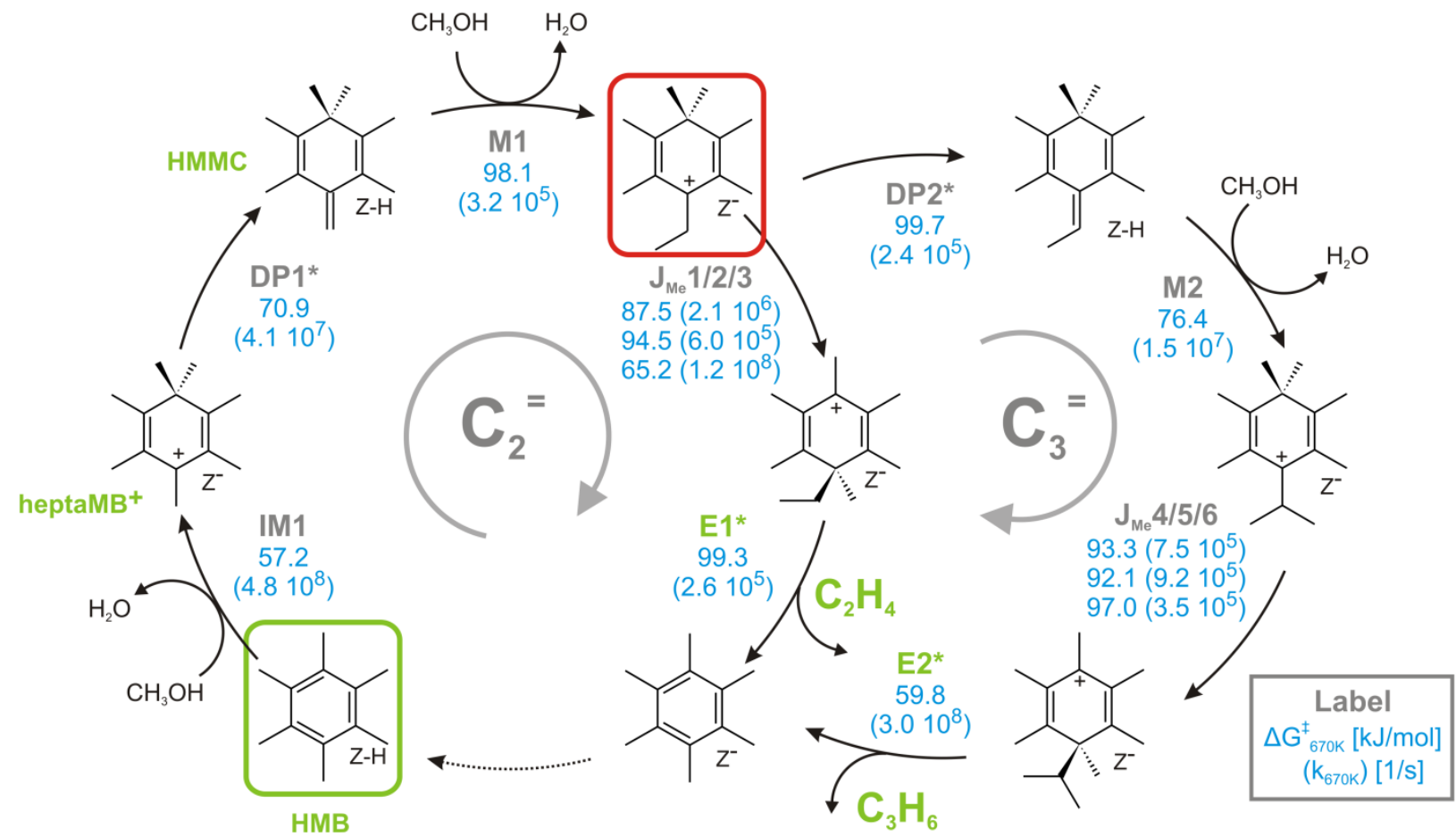

Figure 2. The proposed side-chain mechanism in H-SAPO-34 for ethene $\left(\mathrm{C}_{2}{ }^{=}\right)$and propene $\left(\mathrm{C}_{3}{ }^{=}\right)$formation starting from HMB. Reactions indicated with an asterisk are water assisted. Free energy barriers in $\mathrm{kJ} / \mathrm{mol}$ and intrinsic rate coefficients in $1 / \mathrm{s}$ (in parentheses) at $670 \mathrm{~K}$ are displayed in blue. The products of $\mathrm{E}^{*}{ }^{*}$ and $\mathrm{E} 2^{*}$ still contain a $\mathrm{H}_{3} \mathrm{O}^{+}$cation resulting in the presence of $Z^{-}$at the end of the cycle.

HMB undergoes an ipso methylation IM1, with formation of the gem-methylated heptamethylbenzenium cation (heptaMB ${ }^{+}$). This methylation reaction is known to be a key step in the formation of aliphatic products.[23, 24] Subsequently the heptaMB ${ }^{+}$cation gets deprotonated (DP1) in its para position, as often suggested in literature,[14] with the formation of 1,2,3,3,4,5-hexamethyl-6-methylene-1,4-cyclohexadiene (HMMC). In a next step the exocyclic double bond of HMMC gets methylated (M1). From this point our cycle differs from earlier proposed mechanisms. The gem-methyl group - in para position with respect to the exocyclic double bound - migrates by three relatively fast 1,2-methyl shifts 
$\left(\mathbf{J}_{\mathbf{M e}} \mathbf{1} / \mathbf{2} / \mathbf{3}\right)$ toward the ring carbon whereto the ethyl side-chain is connected. This results in a $\mathrm{sp}^{3}$ hybridized ring carbon atom and consequently weakens the $\mathrm{C}-\mathrm{C}$ bond that needs to be broken for the ethene split-off.[25, 26] The ethene elimination E1 occurs through a concerted mechanism in which simultaneously the $\mathrm{C}-\mathrm{C}$ bond is broken and the terminal methyl group of the ethyl side-chain is deprotonated (see Figure 3a).

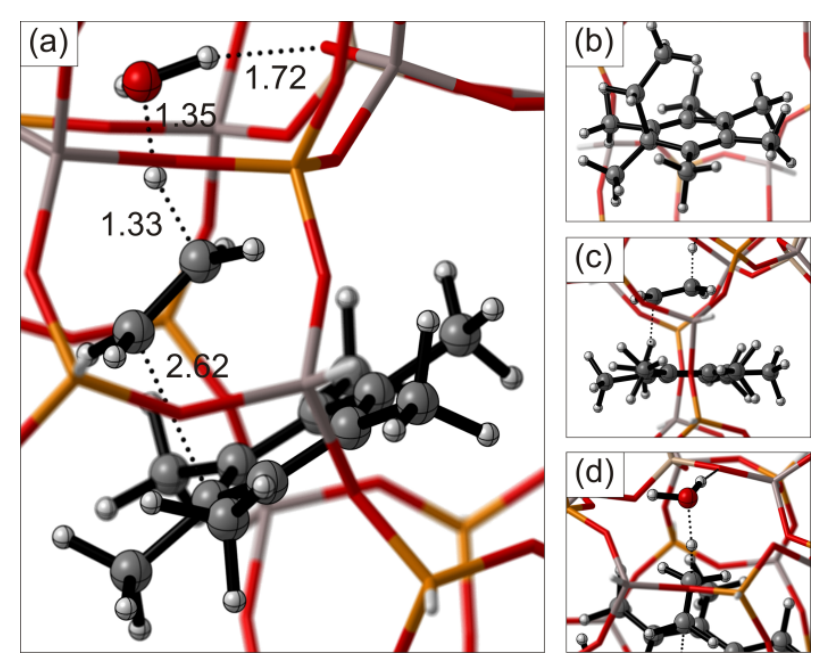

Figure 3. Transition state for the water assisted ethene elimination reaction E1 with distances indicated in $\AA$ (a), the $\mathrm{sp}^{3}$ hybridized ring carbon with ethyl side-chain in the prereactive complex of $\mathbf{E 1}$ (b), the nearly parallel ethyl-HMB complex in the transition state of reaction E1 (c) and the assisting water molecule for the deprotonation during reaction E1 (d).

This last step is assisted by a water molecule, hereby acting as a bridge between the HP molecule and the acid site of the zeolite. During this concerted step ethene is split off and the initial HMB molecule is restored, closing the catalytic cycle. This mechanism was proposed by Arstad et al.[27] and modeled by Chan and Radom on a small 8T cluster.[25] The enthalpic and entropic contributions to the free energy at $670 \mathrm{~K}$ for all reaction steps are listed in Table 1 and activation energies and pre-exponential factors contributing to the reaction rate coefficients are listed in Table S1 of the SI. 
Table 1. Free energy barrier and free energy of reaction with enthalpic and entropic contributions (in $\mathrm{kJ} / \mathrm{mol}$ ) at $670 \mathrm{~K}$ for the different elementary reaction steps in the proposed reaction cycles based on $\mathrm{HMB}$.

\begin{tabular}{ccccccc}
\hline & $\boldsymbol{\Delta G}^{\ddagger}$ & $\boldsymbol{\Delta} \mathbf{H}^{\ddagger}$ & $\mathbf{- T} \boldsymbol{\Delta} \mathbf{S}^{\ddagger}$ & $\boldsymbol{\Delta} \mathbf{G}_{\mathbf{r}}$ & $\boldsymbol{\Delta} \mathbf{H}_{\mathbf{r}}$ & $-\mathbf{T} \boldsymbol{\Delta} \mathbf{S}_{\mathbf{r}}$ \\
\hline $\mathbf{I M 1}$ & 57.2 & 77.6 & -20.4 & 8.1 & 42.2 & -34.0 \\
$\mathbf{D P 1}$ & 70.9 & 50.3 & 20.6 & 30.7 & 26.4 & 4.2 \\
$\mathbf{M 1}$ & 98.1 & 80.9 & 17.2 & -48.2 & -25.3 & -23.0 \\
\hline $\mathbf{J}_{\mathbf{M e}} \mathbf{1}$ & 87.5 & 80.7 & 6.9 & -2.1 & 4.4 & -6.4 \\
$\mathbf{J}_{\mathbf{M e}} \mathbf{2}$ & 94.5 & 85.8 & 8.7 & 38.1 & 31.4 & 6.7 \\
$\mathbf{J}_{\mathbf{M e}} \mathbf{3}$ & 65.2 & 67.6 & -2.4 & -79.7 & -61.8 & 17.9 \\
$\mathbf{E 1}$ & 99.3 & 60.5 & 38.7 & 71.2 & 38.0 & 33.3 \\
\hline $\mathbf{D P 2}$ & 99.7 & 75.3 & 24.4 & 35.9 & 31.7 & 4.2 \\
$\mathbf{M 2}$ & 76.4 & 72.4 & 4.0 & -85.5 & -51.2 & -34.3 \\
$\mathbf{J}_{\mathbf{M e}} \mathbf{4}$ & 93.3 & 84.8 & 8.5 & 13.0 & 10.6 & 2.4 \\
$\mathbf{J}_{\mathbf{M e}} \mathbf{5}$ & 92.1 & 88.0 & 4.1 & 46.8 & 43.9 & 3.0 \\
$\mathbf{J}_{\mathbf{M e}} \mathbf{6}$ & 97.0 & 87.2 & 9.8 & 47.2 & 37.5 & 9.7 \\
$\mathbf{E 2}$ & 59.8 & 33.3 & 26.6 & 15.4 & 3.5 & 12.0 \\
\hline
\end{tabular}

The elimination of ethene, E1, exhibits an intrinsic free energy barrier of $99 \mathrm{~kJ} / \mathrm{mol}$ which is far below the earlier suggested barriers for ethene elimination.[14, 15] This value is composed of an enthalpic barrier of ca. $60 \mathrm{~kJ} / \mathrm{mol}$ and a relatively large entropic barrier of 39 $\mathrm{kJ} / \mathrm{mol}$. The latter value results from the water assistance for the deprotonation. There are three factors that determine the advantageously low barrier of E1 (see Figure 1). Firstly, the $\mathrm{C}-\mathrm{C}$ bond weakens due to the gem-methyl group on the ring carbon wherefrom the ethyl group will be split off (see Figure 3b). Secondly, in the transition state the ethyl side-chain and aromatic ring are nearly parallel, indicating the formation of an alkyl-benzene like complex (see Figure 3c), which is stabilized by non-covalent interactions ${ }^{1} \cdot[22,26,28,29]$ We also modeled a transition state that does not exhibit this particular complex formation and

\footnotetext{
${ }^{1}$ With the M06-2X functional a similar geometry and free energy barrier were found for
} reaction $\mathrm{E} 1$ 
obtained a free energy barrier of $245 \mathrm{~kJ} / \mathrm{mol}$ (Figure S3 and Table S2 of the SI). Finally, the assisting water molecule eases the deprotonation of the ethyl group by facilitating the access to the active site (see Figure 3d). Transition states exhibiting the same reaction coordinate but without an assisting water molecule are characterized by a free energy barrier of $129 \mathrm{~kJ} / \mathrm{mol}$ (Figure S4 and Table S3 of the SI), which is ca. $30 \mathrm{~kJ} / \mathrm{mol}$ higher than the water-assisted transition state, but still lower than all other barriers of mechanistic proposals available in literature.

The intermediate where the ethene and propene producing cycles bifurcate is indicated with a red box in Figure 2. The propene formation route is mechanistically similar to the ethene route, but now the ethyl side-chain in the product of reaction M1 undergoes a second exocyclic deprotonation (DP2) and methylation (M2). After three subsequent 1,2-methyl shifts $\left(\mathbf{J}_{\mathbf{M e}} \mathbf{4 / 5 / 6}\right)$, propene is split off in a concerted step (E2) similar to the ethene formation, whereby an isopropyl-HMB complex is formed in the transition state (Figure S5). Importantly, the rate coefficient for propene elimination is 3 orders of magnitude higher compared to ethene (Figure 2). This difference is due to the formation of a more stable secondary carbenium ion in the transition state involving propene, whereas this is a primary cation during ethene elimination. The corresponding free energy barrier for this reaction is 60 $\mathrm{kJ} / \mathrm{mol}$, being $39 \mathrm{~kJ} / \mathrm{mol}$ lower than for the ethene elimination. This is in line with the experimental observation that in general more propene than ethene is formed during methanol conversion in H-SAPO-34,[7, 13] although care is needed when making conclusions on product selectivity since DP2 exhibits a similar free energy barrier as the ethene elimination step E1 (vide infra). Moreover, it is reported that propene can also be formed during alkene methylation and cracking reactions which clarifies the high initial propene selectivity in $\mathrm{H}$ SAPO-34 as well.[30] 
Apart from the elimination step, some attention is warranted on other elementary steps to compare the proposed results with important experimental or earlier theoretical data. Ipsomethylation IM1 is a fast reaction as HMB is very active toward methylation in H-SAPO34.[8] Deprotonation reaction DP1 is modeled with an assisting water molecule, lowering the free energy barrier by $14 \mathrm{~kJ} / \mathrm{mol}$ compared to the similar reaction without an assisting molecule. Water is sufficiently present inside the chabazite cages as it is a product of all methylation reactions, but also methanol could act as assisting molecule. Our modeling results show that the results for water and methanol assisted deprotonation are nearly identical (Table S4). The main advantage of the assisting molecule is that no additional - and unphysical - rotation of the heptaMB ${ }^{+}$cation is necessary to have access to the Brønsted acid site.[15] Reaction DP1 is endothermic and has an equilibrium constant of $4.010^{-3}$ at $670 \mathrm{~K}$, indicating that in equilibrium conditions the protonated form heptaMB ${ }^{+}$will be preferred (see SI for a further discussion). Bjørgen et al. also reported this observation based on an experimental study in H-Beta.[11] The calculated gas phase proton affinity of the HMMC species is $985 \mathrm{~kJ} / \mathrm{mol}$ which is beyond the threshold values of $874 \mathrm{~kJ} / \mathrm{mol}$ and $820 \mathrm{~kJ} / \mathrm{mol}$ for persistent carbenium ions in H-ZSM-5 and H-Beta as determined by Haw[31] and Svelle and coworkers[32], respectively. It is worth mentioning that the presence of the heptaMB ${ }^{+}$ compound was demonstrated by GC-MS and NMR analysis for MTO conversion in the novel DNL-6 SAPO material, which is related to H-SAPO-34.[33] In the same material, additional ${ }^{13} \mathrm{C}$ methanol experiments indicated the occurrence of a side-chain mechanism.[33] However, for H-SAPO-34 no direct experimental evidence exists for the occurrence of heptaMB ${ }^{+}$nor HMMC. UV/Vis absorption measurements might be very useful, as two absorption bands at 390 and $330 \mathrm{~nm}$ are typically observed during MTO conversion in H-SAPO-34.[34] We calculated absorption energies for the cationic and neutral compound within the H-SAPO-34 
framework and found first singlet transitions at 380 and $312 \mathrm{~nm}$ respectively (Figure S6), meaning that both species contribute to different UV/Vis bands.[35] Further experimental studies with the aim of discriminating between these two compounds under real MTO conditions would help unraveling the reaction mechanism.

The exocyclic methylation M1 is exothermic $\left(\Delta \mathrm{H}_{\mathrm{r}}=-25.3 \mathrm{~kJ} / \mathrm{mol}\right.$ at $\left.670 \mathrm{~K}\right)$ and exhibits a lower rate coefficient than IM1 due to entropic effects (see Tables 1 and S1). Moreover, this is one of the slowest steps in the full catalytic cycle. After three relatively fast methyl shifts, the concerted reaction E1 easily splits off ethene as described above. This finding starkly contrasts with earlier attempts to model this ethene split-off via a side-chain route.[14, 15] Previous investigations reported on highly activated ethene formation via an intramolecular 1,3-hydride shift $[12,14,15]$ or via a two step mechanism with an intermediate spiro structure (routes B and C in Figure S7, respectively).[14, 15] To allow a straightforward comparison, we simulated the earlier reported formation of a spiro structure and found a relatively high free energy barrier at $670 \mathrm{~K}$ of $142.6 \mathrm{~kJ} / \mathrm{mol}$ (Figure S8 and Table S5). The intramolecular 1,3-hydride shift, also referred to as $\beta$-hydride shift, needs to overcome an unfeasible energy barrier of over $200 \mathrm{~kJ} / \mathrm{mol}$.[14, 15] The subtle interplay between various factors makes the newly proposed olefin elimination reaction low-activated, hereby exhibiting a free energy barrier comparable to all other reactions in the presented catalytic cycle (Figure 2, Table 1 and Table S1) ${ }^{2}$

${ }^{2}$ The product of the water assisted ethene elimination as modeled here contains a $\mathrm{H}_{3} \mathrm{O}^{+}$ cation. Modeling the subsequent deprotonation of this hydronium ion yields a slightly negative barrier, being a known phenomenon of the applied cluster methodology for $\mathrm{H}$ SAPO-34 (see Table S6 of the SI) 
After reaction M1 the catalytic cycle bifurcates in an ethene and propene route (red box in Figure 2). For propene formation, deprotonation of the 1-ethyl-2,3,4,4,5,6hexamethylbenzenium cation (DP2) is required. This compound has a slightly higher gas phase proton affinity than HMMC (calculated values of 993 versus $985 \mathrm{~kJ} / \mathrm{mol}$ ), making reaction DP2 energetically less favorable and substantially slower than the deprotonation DP1. The exocyclic methylation (M2) is lower activated and remarkably more exothermic than M1 (see Table 1), because more stable products are formed. Propene is split off in a concerted fashion $(\mathbf{E 2})$ analogous to E1. In the propene elimination route the deprotonation DP2 exhibits the highest free energy barrier.

Next to ethene and propene, also $\mathrm{C}_{4}$ olefins are formed during methanol conversion in $\mathrm{H}$ SAPO-34, hence the formation of isobutene via a similar set of subsequent reactions is studied as well (Tables S1 and S7). Hereby the product of reaction M2 is deprotonated (DP3) and methylated (M3) once more to form an isobutyl side-chain. Again the deprotonation is relatively fast (PA of the involved organic compound is $987 \mathrm{~kJ} / \mathrm{mol}$ ) and the exocyclic methylation $\mathbf{M 3}$ is as fast as reaction M2 but much more exothermic. After three methyl shifts $\left(\mathbf{J}_{\mathbf{M e}} \mathbf{7 / 8 / 9}\right)$ the isobutyl side-chain is already partially disconnected from the HMB molecule (Figure S9). No further energy maximum along the breaking C-C bond could be located, indicating that the last methyl shift actually produces the isobutyl cation which can deprotonate or isomerize toward linear $\mathrm{C}_{4}$ products.

\section{Conclusions}

This first principle kinetic study shows that a low-barrier path for olefin formation in $\mathrm{H}$ SAPO-34 is now found. The reaction cycle is based on the earlier proposed side-chain mechanism starting from HMB. The low free energy barrier for ethene formation results from 
an $\mathrm{sp}^{3}$ carbon center of the organic intermediate, the formation of an alkyl-benzene complex and the assistance of water in the transition state. Propene can be split off in a similar fashion. For ethene formation, the elimination reaction exhibits the highest free energy barrier and lowest rate coefficient, whereas this is the second deprotonation reaction in the route toward propene. The results reported in this work may be regarded as a breakthrough in unraveling the MTO process over H-SAPO-34 catalysts as no plausible path has been reported so far. This work underlines the current power of ab initio simulations to provide detailed insight into reaction mechanisms and intermediates. It gives an impulse for further experimental and theoretical studies to elucidate the governing reaction cycles during olefin production in $\mathrm{H}$ SAPO-34 and other MTO catalysts. Further studies can focus on the accurate prediction of $\mathrm{C}_{2^{-}}, \mathrm{C}_{3^{-}}$, and $\mathrm{C}_{4}$-selectivities, wherefore a first step is taken in this work. As new MTO catalysts are being tested nowadays, experimental set-ups can be pointed toward the characterization of the computationally suggested reaction intermediates. In this view, calculated UV/Vis spectra for some important intermediates are reported in this work. More in general, the validity of the reaction path should be explored in other materials such as $\mathrm{H}$ ZSM-5 for which the side-chain mechanism was earlier found to be highly activated. Additionally, the influence of the characteristics of the initial HP compound and the catalyst acidity could be tested.

\section{ACKNOWLEDGMENT}

We are grateful to the Research Foundation - Flanders (FWO), the Research Board of Ghent University, and BELSPO in the frame of IAP P7/05. Funding was also received from the European Research Council under the European Community's Seventh Framework Program [FP7(2007-2013) ERC grant agreement number 240483]. Computational resources 
and services used in this work were provided by the Stevin Supercomputer Infrastructure of Ghent University.

\section{REFERENCES}

[1] U. Olsbye, S. Svelle, M. Bjorgen, P. Beato, T.V.W. Janssens, F. Joensen, S. Bordiga, and K.P. Lillerud, Angew. Chem., Int. Edit. 51 (2012) 5810-5831.

[2] S. Ilias, and A. Bhan, ACS Catal. (2013) 18-31.

[3] K. Hemelsoet, J. Van der Mynsbrugge, K. De Wispelaere, M. Waroquier, and V. Van Speybroeck, ChemPhysChem (2013) DOI:10.1002/cphc.201201023

[4] J.Q. Chen, A. Bozzano, B. Glover, T. Fuglerud, and S. Kvisle, Catal. Today 106 (2005) 103-107.

[5] J.F. Haw, and D.M. Marcus, Top. Catal. 34 (2005) 41-48.

[6] W.G. Song, J.F. Haw, J.B. Nicholas, and C.S. Heneghan, J. Am. Chem. Soc. 122 (2000) 10726-10727.

[7] B.P.C. Hereijgers, F. Bleken, M.H. Nilsen, S. Svelle, K.P. Lillerud, M. Bjorgen, B.M. Weckhuysen, and U. Olsbye, J. Catal. 264 (2009) 77-87.

[8] V. Van Speybroeck, K. Hemelsoet, K. De Wispelaere, Q. Qian, J. Van der Mynsbrugge, B. De Sterck, B.M. Weckhuysen, and M. Waroquier, ChemCatChem 5 (2013) 173-184.

[9] W.G. Song, H. Fu, and J.F. Haw, J. Phys. Chem. B 105 (2001) 12839-12843.

[10] B. Arstad, and S. Kolboe, J. Am. Chem. Soc. 123 (2001) 8137-8138.

[11] M. Bjorgen, U. Olsbye, D. Petersen, and S. Kolboe, J. Catal. 221 (2004) 1-10.

[12] B. Arstad, J.B. Nicholas, and J.F. Haw, J. Am. Chem. Soc. 126 (2004) 2991-3001.

[13] W.G. Song, H. Fu, and J.F. Haw, J. Am. Chem. Soc. 123 (2001) 4749-4754.

[14] C.M. Wang, Y.D. Wang, Z.K. Xie, and Z.P. Liu, J. Phys. Chem. C 113 (2009) 45844591.

[15] D. Lesthaeghe, A. Horre, M. Waroquier, G.B. Marin, and V. Van Speybroeck, Chem.- Eur. J. 15 (2009) 10803-10808.

[16] M. J. Frisch et al., Gaussian 09, revision B.01 ed.; Gaussian, Inc.: Wallingford CT (2009).

[17] S. Grimme, J. Antony, S. Ehrlich, and H. Krieg, J. Chem. Phys. 132 (2010) 19.

[18] V. Van Speybroeck, J. Van der Mynsbrugge, M. Vandichel, K. Hemelsoet, D. Lesthaeghe, A. Ghysels, G.B. Marin, and M. Waroquier, J. Am. Chem. Soc. 133 (2011) 888-899.

[19] S. Svelle, C. Tuma, X. Rozanska, T. Kerber, and J. Sauer, J. Am. Chem. Soc. 131 (2009) 816-825.

[20] J. Van der Mynsbrugge, K. Hemelsoet, M. Vandichel, M. Waroquier, and V. Van Speybroeck, J. Phys. Chem. C 116 (2012) 5499-5508.

[21] A. Ghysels, T. Verstraelen, K. Hemelsoet, M. Waroquier, and V. Van Speybroeck, J. Chem Inf. Model. 50 (2010) 1736-1750.

[22] S. Kolboe, J. Phys. Chem. A 115 (2011) 3106-3115.

[23] M. Bjorgen, U. Olsbye, and S. Kolboe, J. Catal. 215 (2003) 30-44.

[24] D. Lesthaeghe, B. De Sterck, V. Van Speybroeck, G.B. Marin, and M. Waroquier, Angew. Chem., Int. Edit. 46 (2007) 1311-1314.

[25] B. Chan, and L. Radom, Can. J. Chem. 88 (2010) 866-876.

[26] S. Kolboe, S. Svelle, and B. Arstad, J. Phys. Chem. A 113 (2009) 917-923.

[27] B. Arstad, S. Kolboe, and O. Swang, J. Phys. Chem. B 108 (2004) 2300-2308. 
[28] S. Kolboe, and S. Svelle, J. Phys. Chem. A 112 (2008) 6399-6400.

[29] S. Kolboe, J. Phys. Chem. A 116 (2012) 3710-3716.

[30] J.Z. Li, Y.X. Wei, G.Y. Liu, Y. Qi, P. Tian, B. Li, Y.L. He, and Z.M. Liu, Catal. Today 171 (2011) 221-228.

[31] J.F. Haw, Phys. Chem. Chem. Phys. 4 (2002) 5431-5441.

[32] S. Svelle, and M. Bjorgen, J. Phys. Chem. A 114 (2010) 12548-12554.

[33] J.Z. Li, Y.X. Wei, J.R. Chen, P. Tian, X. Su, S.T. Xu, Y. Qi, Q.Y. Wang, Y. Zhou, Y.L. He, and Z.M. Liu, J. Am. Chem. Soc. 134 (2012) 836-839.

[34] W.L. Dai, M. Scheibe, N.J. Guan, L.D. Li, and M. Hunger, ChemCatChem 3 (2011) 1130-1133.

[35] Y.J. Jiang, J. Huang, V.R.R. Marthala, Y.S. Ooi, J. Weitkamp, and M. Hunger, Microporous Mesoporous Mater. 105 (2007) 132-139. 